\title{
Abnormal increase of neuronal precursor cells and exacerbated neuroinflammation in the corpus callosum in murine model of systemic lupus erythematosus
}

\author{
Joseph Wai-Hin Leung ${ }^{\mathrm{a}, 1}$, Benson Wui-Man Lau ${ }^{\mathrm{b}, 1}$, Vera Sau-Fong Chan ${ }^{\mathrm{a}}$, Chak-Sing Lau ${ }^{\mathrm{a}, *}$ \\ and Kwok-Fai $\mathrm{So}^{\mathrm{c}, \mathrm{d}, \mathrm{e}, \mathrm{f}, \mathrm{g}, 2, *}$ \\ ${ }^{a}$ Department of Medicine, LKS Faculty of Medicine, The University of Hong Kong, Hong Kong \\ ${ }^{\mathrm{b}}$ Rehabilitation Sciences, The Hong Kong Polytechnic University, Hong Kong \\ ${ }^{\mathrm{c}}$ Department of Ophthalmology, LKS Faculty of Medicine, The University of Hong Kong, Hong Kong \\ ${ }^{\mathrm{d}}$ State Key Laboratory of Brain and Cognitive Science, The University of Hong Kong, Hong Kong \\ ${ }^{\mathrm{e}}$ GHM Institute of CNS Regeneration, Jinan University, Guangzhou, China \\ ${ }^{\mathrm{f}}$ Co-innovation Center of Neuroregeneration, Nantong University, Jiangsu, China \\ ${ }^{\mathrm{g}}$ Ministry of Education CNS Regeneration International Collaborative Joint Laboratory, Jinan University, \\ Guangzhou, China
}

\begin{abstract}
.
Purpose: Systemic Lupus Erythematosus (SLE) is an autoimmune disease which is characterised by elevated levels of autoantibodies and cytokines in the body. Via alteration of the regulation of inflammation, damage to different organ systems, including the central nervous system (CNS), was found in SLE patients. Patients diagnosed with SLE were reported to suffer from different kinds of psychiatric signs and symptoms. As neurogenesis has been suggested to be a potential key player of psychiatric symptoms and emotional behavior disturbances, this study aims to investigate whether neurogenesis is altered in an animal model of SLE. Also, neuroinflammation was studied.

Methods: Female NZB/W F1 mice were used as an animal model of SLE. Animals were divided into two groups: 1. prediseased mice (lupus-prone NZB/W F1 female mice, age 10-15 weeks, negative for proteinuria and with basal levels of serum anti-dsDNA autoantibodies) and 2. diseased mice (NZB/W F1 female mice, $>25$ weeks of age, with elevated serum levels of anti-dsDNA autoantibodies and with persistent proteinuria of $>3 \mathrm{mg} / \mathrm{ml}$ for more than 2 weeks). Comparisons of the levels of neurogenesis and neuroinflammtion between two groups of mice were studied by the immunohistochemistry.

Results: After the onset of SLE symptoms, a reduction of neurogenesis in the hippocampus was found, while there was a dramatic increase of doublecortin (DCX+) neuronal precursor cells in the corpus callosum (CC) and in the subventricular zone (SVZ). Meanwhile, exacerbated inflammation was present in the corpus callosum of the diseased mice, which was suggested by the increased number of GFAP+ cells and IBA-1+ cells.

Conclusion: To the best of our knowledge, this is the first study showing an increase of neuronal precursor cells in the corpus callosum of the female NZB/W F1 mice. The present study suggests a coincidence but not a causal relationship
\end{abstract}

\footnotetext{
${ }^{1}$ Co-first authors.

${ }^{2}$ Co-corresponding authors.

*Corresponding author: Kwok-Fai So, Department of Ophthalmology, The University of Hong Kong, 5 Sassoon Road, Hong
}

Kong. Tel.: +852 2831 5366; Fax: +852 2817 0941; E-mail: hrmaskf@hku.hk. 
between neurogenesis and neuroinflammation. The present results have also provided new insight showing that the altered neurogenesis and neuroinflammation may be a potential neurological mechanism for the cognitive and mood disturbance found in the SLE patients.

Keywords: Neurogenesis, neuroinflammation, systemic lupus erythematosus, corpus callosum, subventricular zone, doublecortin, GFAP, IBA-1

\section{Introduction}

Systemic lupus erythematosus (SLE) is an autoimmune disorder featured with production of a variety of autoantibodies (C. S. Lau, Yin, \& Mok, 2006). This disease is more commonly found in females than in males with a ratio 9:1 (D'Cruz, Khamashta, \& Hughes, 2007). Although the pathogenesis of SLE remains obscure, it has been suggested that genetic (D'Cruz et al., 2007; Morel et al., 2000; Sestak, Furnrohr, Harley, Merrill, \& Namjou, 2011), epidemiologic (Cooper et al., 2004; Parks et al., 2002) and hormonal factors (Manson \& Isenberg, 2003) are involved. The clinical manifestation of SLE involves multiple organ systems and the central nervous system (CNS) is one of them. Different kinds of brain abnormalities, including hippocampal and corpus callosum atrophy as well as reduction of cortical thickness have been reported from the SLE patients (Appenzeller, Carnevalle, Li, Costallat, \& Cendes, 2006; Appenzeller, Rondina, Li, Costallat, \& Cendes, 2005; Jung et al., 2010). Also, patients with neuropsychiatric SLE (NPSLE) were found to have neuropsychiatric symptoms such as cognitive dysfunction, delirium (acute confusion state), anxiety, mood disturbance and psychosis (Stojanovich, Zandman-Goddard, Pavlovich, \& Sikanich, 2007), which may impact negatively on quality of life (Chen, Pan, Hsiung, \& Chung, 2015). Although the mechanisms underlying the psychiatric symptoms remain elusive, it has been shown that SLE could lead to damage in the hippocampus (Ballok, Woulfe, Sur, Cyr, \& Sakic, 2004) and the anti-NMDAR antibody presented in SLE patients was able to cause neuronal death (Kowal et al., 2006). Given that hippocampus is involved in emotional regulation, mood disturbances in SLE patients may be a possible consequence of hippocampal damage.

Adult neurogenesis is the process in which functional neurons are generated from their adult progenitor cells (Ming \& Song, 2005, 2011). The subventricular zone (SVZ) is one of the brain regions where active neurogenesis takes place (Ming \& Song, 2005, 2011). New neurons generated in the SVZ migrate to the olfactory bulb (OB), in where they become mature and differentiate into interneurons (Altman, 1969; Kornack \& Rakic, 2001; Lois \& Alvarez-Buylla, 1994). The SVZ neurogenesis was suggested to be important for odor discrimination (Alvarez-Buylla \& Garcia-Verdugo, 2002; Gheusi et al., 2000) and sexual behaviour (Bonfanti, Peretto, Merighi, \& Fasolo, 1997; Smith, Pencea, Wang, Luskin, \& Insel, 2001). Apart from the SVZ, adult neurogenesis also occurs in the hippocampus (Ming \& Song, 2005, 2011). Hippocampal neurogenesis may enhance synaptic plasticity within a specific time period (Ge, Yang, Hsu, Ming, \& Song, 2007) and it is necessary for pattern separation (Clelland et al., 2009). Altered hippocampal neurogenesis is also suggested to be a possible mechanism underlying psychiatric symptoms (Ruan et al., 2014). Neurogenesis has been found to increase under different pathological conditions, including stroke (Arvidsson, Collin, Kirik, Kokaia, \& Lindvall, 2002; Liu, Solway, Messing, \& Sharp, 1998; Parent, Vexler, Gong, Derugin, \& Ferriero, 2002), intracerebral hemorrhage (Masuda et al., 2007), mechanical brain injury (SundholmPeters, Yang, Goings, Walker, \& Szele, 2005), Huntington's disease (Curtis et al., 2003) and seizureinduced injury (Parent, Valentin, \& Lowenstein, 2002). Studies have suggested that neurogenesis may be a particular form of endogenous neural repair in response to neuronal cell loss caused by the diseases. Brain damage and neuropsychiatric symptoms have been reported from the SLE patients; however, study focusing on neurogenesis and SLE is limited.

Doublecortin (DCX) is a microtubule-associated protein which is expressed in neuronal precursor during brain development (des Portes et al., 1998; Gleeson, Lin, Flanagan, \& Walsh, 1999). Its expression peaks at the regions where active neurogenesis occurs, including the dentate gyrus and the lateral ventricle (Brown et al., 2003), but it is unlikely to be found in the non-neurogenic regions (Nacher, Crespo, \& McEwen, 2001). It has been shown that DCX is specifically expressed by the newly generated healthy neurons in the adult dentate gyrus (Rao \& Shetty, 2004) and there is also evidence suggesting that DCX 
is a reliable and specific marker for measuring the rate of neurogenesis (Couillard-Despres et al., 2005). Previous studies have reported cell proliferation could be found in the SVZ of the MRL/MpJ mice and the MRL/MpJ-Fas ${ }^{l p r}$ mice (Baker et al., 2006; Stanojcic, Burstyn-Cohen, Nashi, Lemke, \& Sakic, 2009). The present study used DCX as the marker of neuronal precursor cells to study whether neurogenesis could be found in our SLE animal model- female NZB/W F1 mice; also we would like to investigate whether new neuronal cells could be observed in the brain regions other than the SVZ.

In addition to neurogenesis, neuroinflammatory responses after the onset of SLE symptoms were also examined in the present study. Neuroinflammation is usually characterised by microglial and astroglial activation. Microglia are hallmark of brain pathology (Dheen, Kaur, \& Ling, 2007) and these cells respond to different types of CNS injuries (Gehrmann, Matsumoto, \& Kreutzberg, 1995). During autoimmune inflammation, microglia release different kinds of cytokines such as IL- 1 , TNF- $\alpha$ and IFN- $\gamma$ and other harmful chemical species, including proteases and reactive oxygen intermediates (Gehrmann et al., 1995). The neuronal cell injury has been shown to be mediated by the neurocytotoxic-free radical secreted by the microglia, e.g. nitric oxide (NO) (Banati, Gehrmann, Schubert, \& Kreutzberg, 1993; Chao, Hu, Molitor, Shaskan, \& Peterson, 1992). Being similar to microglia, astrocytes could also vigorously react to neurodegenerative stimuli (Eng \& Ghirnikar, 1994) and their activation is considered as an implication of the pathogenesis of various neurodegenerative diseases (Eng \& Ghirnikar, 1994). It was suggested that severe astroglial activation could damage the brain by the initiation of inflammatory responses (Tani et al., 1996), and astrocytes could produce toxic substances such as nitrite oxide which causes neuronal cell death (Stewart, Sharpe, Clark, \& Heales, 2000). The marker widely used for the detection of microglia and astrocytes are respectively ionized calcium binding adaptor molecule 1 (IBA-1) and glial fibrillary acidic protein (GFAP). IBA-1 is a protein which is specifically expressed by the microglia and it could not be detected in neurons, astroglia, or oligodendroglia (Ito et al., 1998), whereas GFAP is usually expressed by astrocytes and its expression has been reported to correspond to the level of astroglial activation (Eng \& Ghirnikar, 1994; Eng, Yu, \& Lee, 1992). To study SLE neuroinflammation in the present study, we examined both IBA-1+ cells and GFAP+ cells in our SLE animal model.

\section{Materials and methods}

\subsection{Animals}

Female NZB/W F1 mice were used as an animal model of SLE since they develop SLE symptoms spontaneously (Perry, Sang, Yin, Zheng, \& Morel, 2011). In the present study, they were divided into two groups: Pre-diseased mice $(n=5-8)$ and diseased mice $(n=4-8)$. Pre-diseased mice were usually 10-15 weeks old, before the onset of SLE symptoms; diseased mice were usually older than 25 weeks and had SLE symptoms. Disease progression was assessed by weekly monitoring of two parameters: Serum anti-dsDNA IgG in arbitrary unit (by ELISA) and proteinuria measurement (by protein dipstick). Pre-diseased mice were negative for proteinuria with basal levels of anti-dsDNA autoantibodies. Diseased mice had serum titre $>3$ standard deviations (SD) above the mean anti-dsDNA autoantibodies titre of the pre-diseased mice and had persistent proteinuria ( $>3 \mathrm{mg} / \mathrm{ml}$ for more than 2 weeks). The experimental protocol was approved by the animal ethics committee of The University of Hong Kong (Committee on the Use of Live Animals in Teaching and Research). Animals were housed with a 12-h light/ dark cycle.

\subsection{Tissue processing and immunohistochemistry}

Brains of the mice were dissected and fixed in $4 \%$ paraformaldehyde overnight. Cryosections of $40 \mu \mathrm{m}$ thickness were further prepared in 1-in-12 series by using freezing microtome.

To detect doublecortin (DCX), brain sections were affixed to gelatine-coated slides and allowed to air dry overnight. For antigen retrieval, sections were incubated in citric acid (0.01M, pH6.0) at $90^{\circ} \mathrm{C}$ for 10 minutes. After blocking with $10 \%$ goat serum, the sections were incubated with primary antibody (rabbit anti-DCX antibody, 1:400 dilution, Cell Signaling Technology) overnight at room temperature. Then the sections were incubated with secondary antibody (biotinylated goat anti-rabbit antibody, 1:200 dilution, Dako) at room temperature for 2 hours and they were further incubated with avidin-biotin complex (ABC) solution (1:200, Vector laboratories) at room temperature for 2 hours. DCX staining was visualised by liquid DAB substrate kit (Invitrogen) according to the manufacturer's protocol. After counterstaining with eosin and dehydrating through graded alcohols, sections were cleared in xylene and mounted. 
For the detection of ionized calcium binding adaptor molecule-1 (IBA-1) and glial fibrillary acidic protein (GFAP), brain sections on the gelatine-coated slides were blocked by $10 \%$ goat serum after incubation in citric acid $(0.01 \mathrm{M}, \mathrm{pH} 6.0)$ at $90^{\circ} \mathrm{C}$ for 10 minutes for the antigen retrieval. Brain sections were then incubated with primary antibody (rabbit anti-IBA-1 antibody, 1:500 dilution, Wako) or primary antibody (rabbit anti-GFAP antibody, 1:1000 dilution, Dako) overnight at room temperature. Secondary antibody (Alexa fluor 488-conjugated goat anti-rabbit antibody, 1:200 dilution, Invitrogen) was used for both IBA-1 and GFAP staining.

Stereoinvestigator program (MicroBrightField, Williston, VT) was used to quantify the number of antibody positive cells. (B.W. Lau et al., 2011). All the quantification processes were conducted in a blinded manner. Results were expressed as number of cells per section for the corpus callosum, the SVZ and the dentate gyrus.

\subsection{Statistical analysis}

Comparisons between two groups of mice were performed by Student's $t$-test. Statistically significant difference was indicated when $p \leq 0.05$. Data are expressed as mean \pm SEM.

\section{Results}

\subsection{Change in brain colour after the onset of SLE symptoms}

When compared to the brains of the pre-diseased mice, the brains of the diseased mice appeared paler upon dissection (Fig. 1). No perfusion process was performed on the brains of both groups of mice.

\subsection{Significant increase in $D C X+$ neuronal precursor cells in the corpus callosum and the SVZ after the onset of SLE symptoms}

To study neurogenesis before and after the onset of SLE symptoms, we used DCX immunohistochemistry to compare the number of neuronal precursor cells in the pre-diseased mice and the diseased mice. The results showed that significantly higher numbers of DCX + cells were found in the corpus callosum of the diseased mice when compared to the pre-diseased mice (Fig. 2; $p<0.05$ ). After finding neuronal precursor cells increased dramatically in the corpus

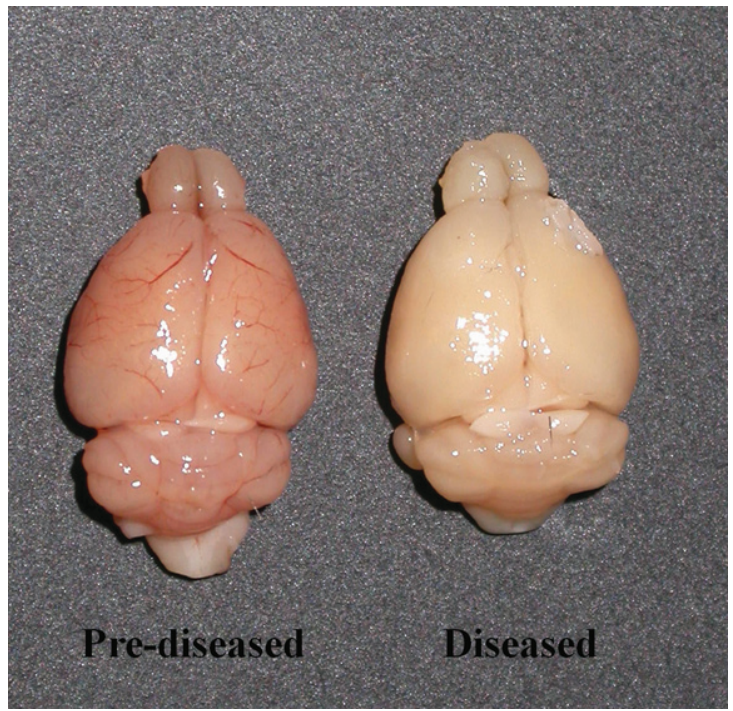

Fig. 1. Unfixed and non-perfused brains of the pre-diseased mice and the diseased mice. Photograph showing the brains of the prediseased mice (left) and the diseased mice (right).

callosum after the onset of SLE symptoms, we further quantified DCX+ cells in the SVZ and the cortex in both groups of mice. The number of DCX+cells in the SVZ was significantly higher in the diseased mice when compared to the pre-diseased mice (Fig. 2; $p<0.05)$. In the cortex, no DCX + cells could be found in either group (Fig. 3). As the DCX+cells in the corpus callosum of our SLE animal model exhibited migrating-like phenotype (Fig. 4), it was likely that the DCX+cells found in the diseased mice were migrating.

\subsection{Decrease in $D C X+$ neuronal precursor cells in the dentate gyrus after the onset of SLE symptoms}

We studied whether neurogenesis was altered in the hippocampus after the onset of SLE symptoms. In contrast to the corpus callosum and the SVZ, we found that the number of DCX+cells in the dentate gyrus of the diseased mice significantly decreased when compared to the pre-diseased mice (Fig. 5; $p<0.01)$.

\subsection{Increase in IBA-1+cells and GFAP+cells in the corpus callosum after the onset of SLE symptoms}

Apart from DCX+cells, we examined IBA$1+$ cells and GFAP+cells in the corpus callosum 

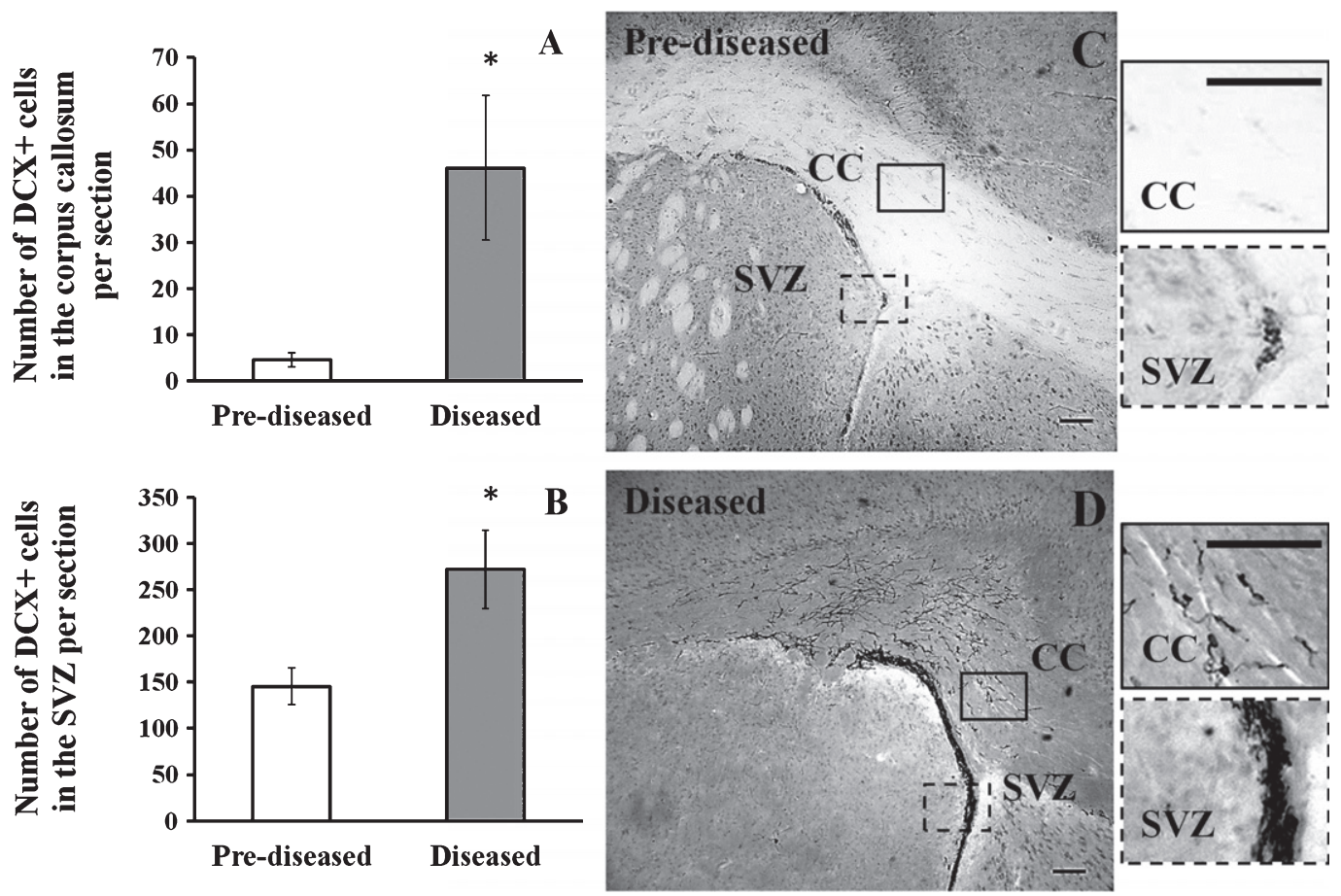

Fig. 2. DCX+cells in the corpus callosum and the SVZ of the pre-diseased mice and the diseased mice (A) Quantification of DCX+cells in the corpus callosum of the pre-diseased mice and the diseased mice. Results were expressed as mean \pm SEM., $n=5$ for each group of mice. Data was analysed by Student's $t$-test. ${ }^{*} p<0.05$ compared with the pre-diseased mice. (B) Quantification of DCX+cells in the SVZ of the pre-diseased mice and the diseased mice. Results were expressed as mean \pm SEM., $n=7$ for the pre-diseased mice and $n=8$ for the diseased mice. Data was analysed by Student's $t$-test. ${ }^{*} p<0.05$ compared with the pre-diseased mice. (C) Representative image showing DCX+cells in the corpus callosum and the SVZ of the pre-diseased mice; scale bar $100 \mu \mathrm{m}$. (D) Representative image showing DCX+cells in the corpus callosum and the SVZ of the diseased mice; scale bar: $100 \mu \mathrm{m}$.

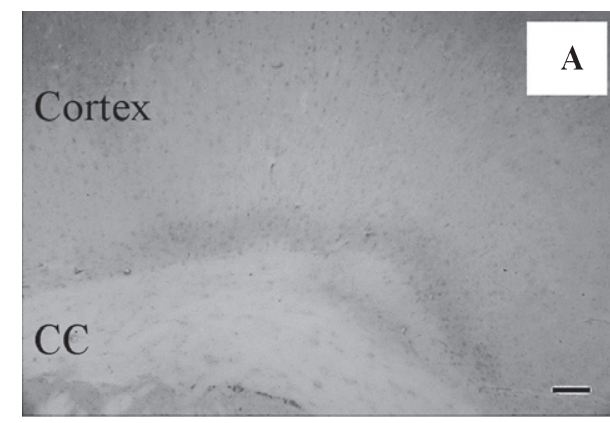

Pre-diseased

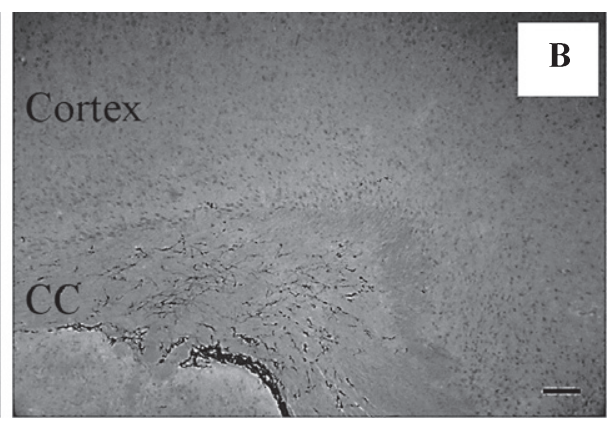

Diseased

Fig. 3. No DCX+cell was found in the cortex of the pre-diseased mice and the diseased mice Representative images showing no DCX+cell was found in the cortex of (A) the pre-diseased mice and (B) the diseased mice; scale bar: $100 \mu \mathrm{m}$.

of the pre-diseased mice and the diseased mice. The results showed that the number of IBA- $1+$ cells (Fig. 6; $p<0.01$ ) and GFAP+cells (Fig. 7; $p<0.05$ ) was significantly higher in the diseased mice when compared to the pre-diseased mice.

\section{Discussion}

The major finding of the present study is that there was a dramatic increase of DCX+cells detected in the corpus callosum of the female NZB/W F1 

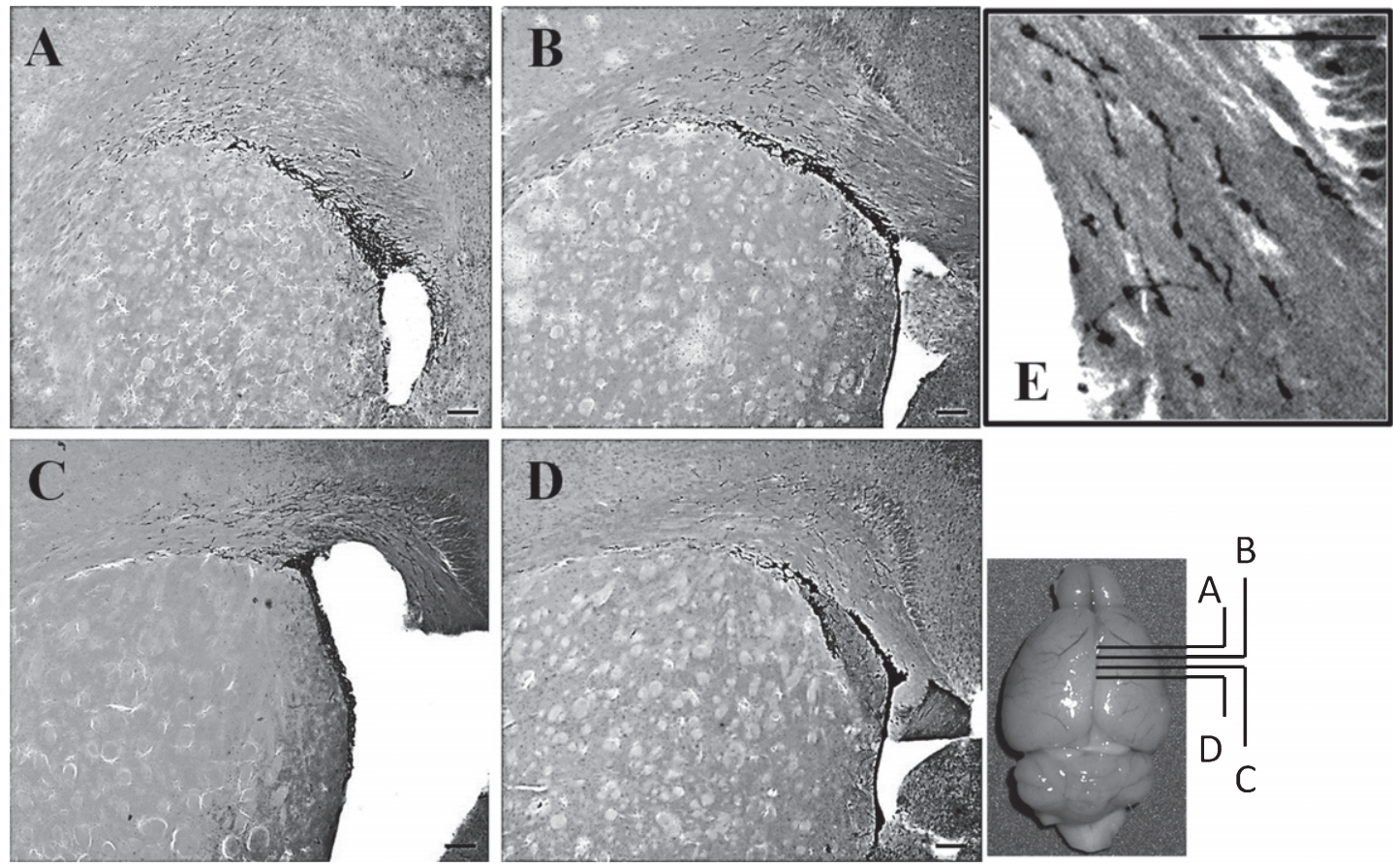

Fig. 4. DCX+ cells in the corpus callosum and the SVZ after the onset of SLE symptoms Photos taken from anterior to posterior brain sections showing the distribution of DCX+cells in the corpus callosum and the SVZ of the diseased mice; scale bar: $100 \mu \mathrm{m}$. The DCX+cells exhibited the migrating-like phenotype and it was shown in the magnified photo (Fig. 4E); scale bar: $100 \mu \mathrm{m}$.
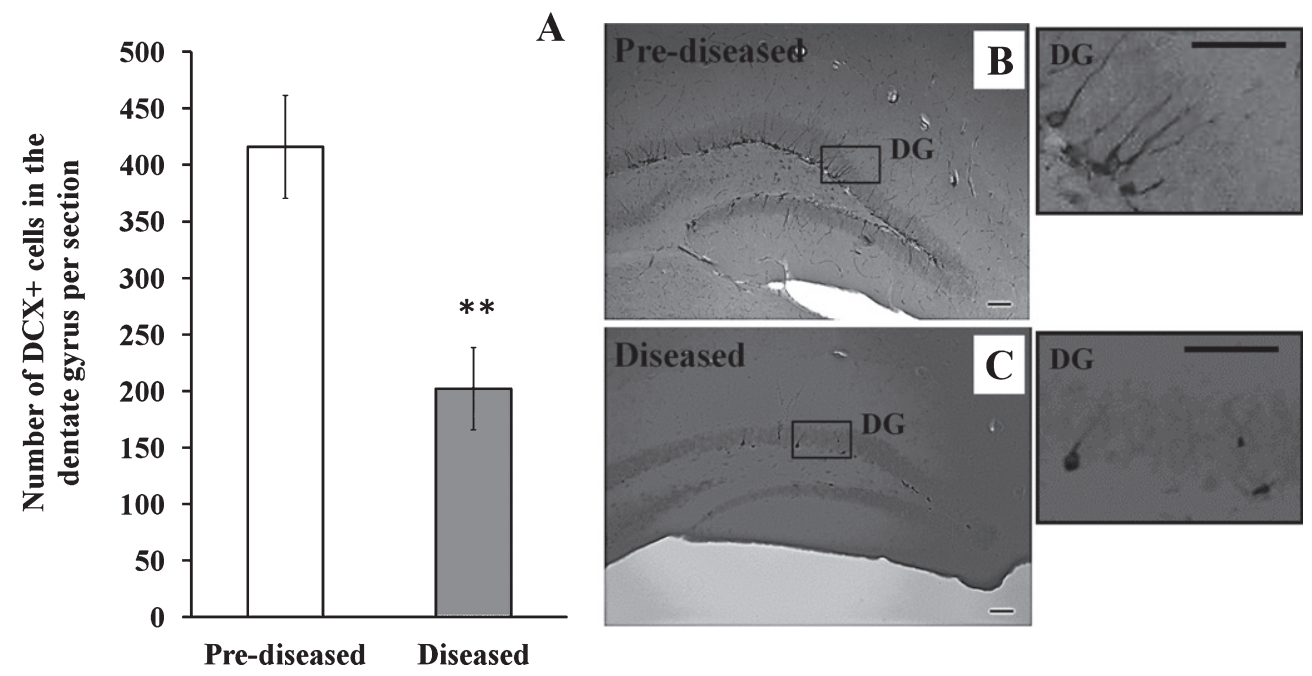

Fig. 5. DCX+ cells in the dentate gyrus of the pre-diseased mice and the diseased mice (A) Quantification of DCX+cells in the dentate gyrus of the pre-diseased mice and the diseased mice. Results were expressed as mean \pm SEM., $n=5$ for the pre-diseased mice and $n=4$ for the diseased mice. Data was analysed by Student's $t$-test. ${ }^{* *} p<0.01$ compared with the pre-diseased mice. (B) Representative image showing $\mathrm{DCX}+$ cells in the dentate gyrus of the pre-diseased mice; scale bar $50 \mu \mathrm{m}$. (C) Representative image showing DCX+cells in the dentate gyrus of the diseased mice; scale bar: $50 \mu \mathrm{m}$.

mice after the onset of SLE symptoms with significant increases also in the SVZ; in addition, we have found reduced neurogenesis in the hippocampus in our SLE animal model (Table 1). Consistent with our results, suppressed neurogenesis has been reported in the previous study in another animal model of SLEBAFF mice, in which a smaller number of hippocampal DCX+cells were found when compared to the 


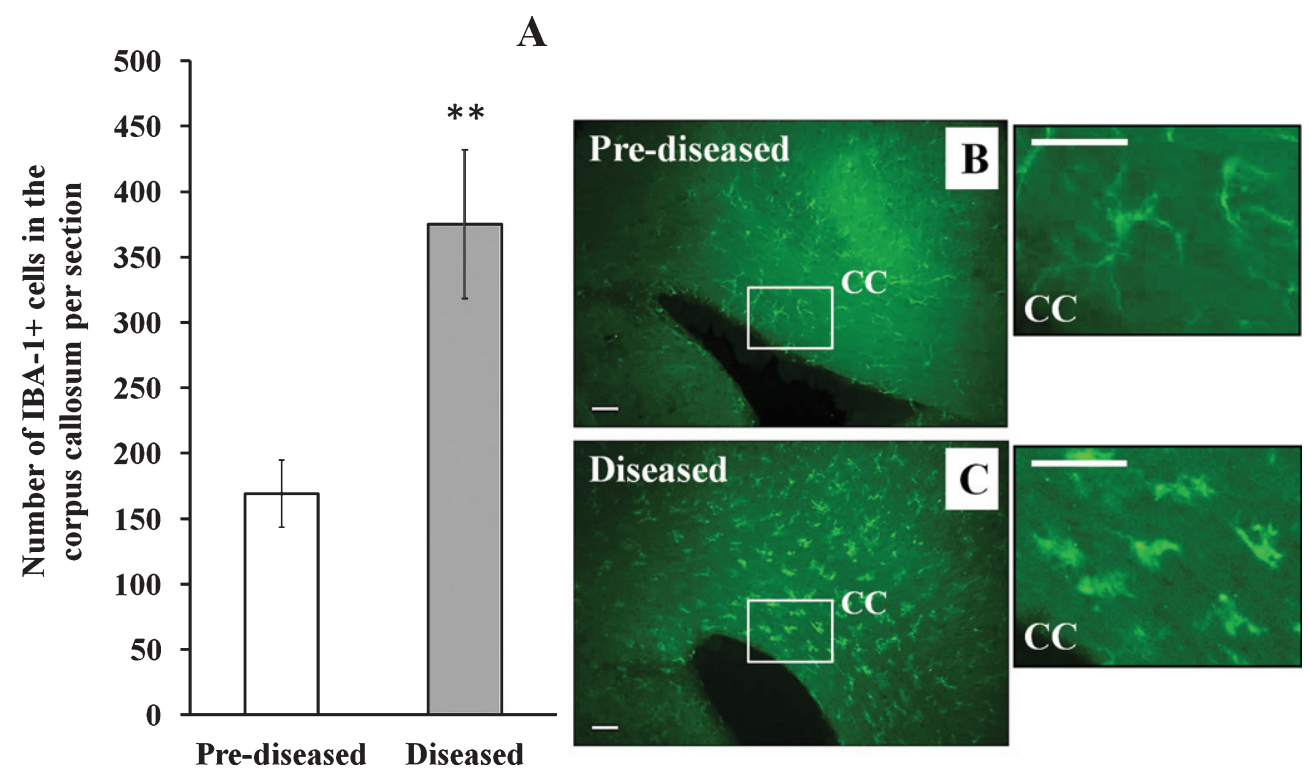

Fig. 6. IBA-1+ cells in the corpus callosum of the pre-diseased mice and the diseased mice (A) Quantification of IBA-1+cells in the corpus callosum of the pre-diseased mice and the diseased mice. Results were expressed as mean \pm SEM., $n=8$ for each group of mice. Data was analysed by Student's $t$-test. ${ }^{* *} p<0.01$ compared with the pre-diseased mice. (B) Representative immunofluorescent image showing IBA-1+ cells in the corpus callosum of the pre-diseased mice; scale bar: $100 \mu \mathrm{m}$. (C) Representative immunofluorescent images showing IBA- $1+$ cells in the corpus callosum of the diseased mice; scale bar: $100 \mu \mathrm{m}$.

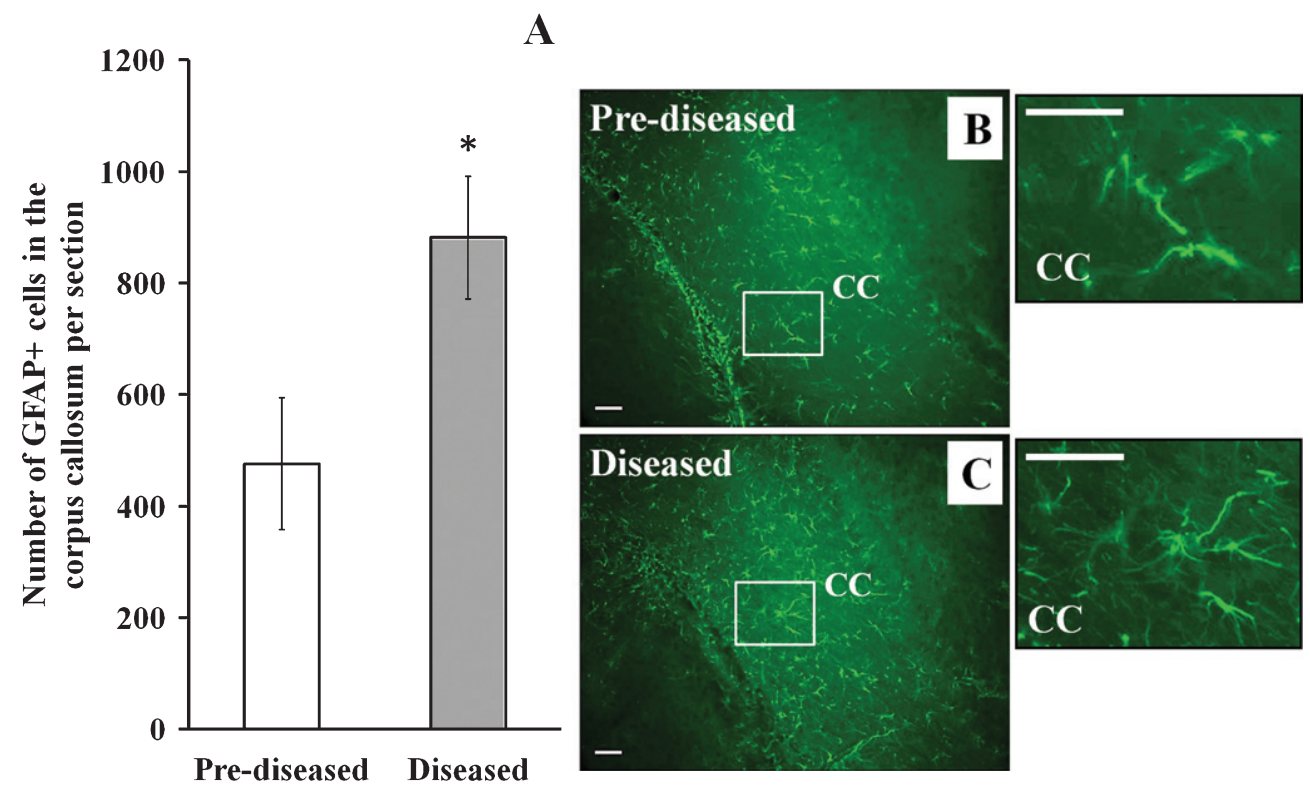

Fig. 7. GFAP+ cells in the corpus callosum of the pre-diseased mice and the diseased mice (A) Quantification of GFAP+cells in the corpus callosum of the pre-diseased mice and the diseased mice. Results were expressed as mean \pm SEM., $n=7$ for the pre-diseased mice and $n=6$ for the diseased mice. Data was analysed by Student's $t$-test. ${ }^{*} p<0.05$ compared with the pre-diseased mice. (B) Representative immunofluorescent image showing GFAP+cells in the corpus callosum of the pre-diseased mice; scale bar: $50 \mu \mathrm{m}$. (C) Representative immunofluorescent image showing GFAP+ cells in the corpus callosum of the diseased mice; scale bar: $50 \mu \mathrm{m}$.

wild type (Crupi et al., 2010) (Table 2). Our study has further suggested the relationship between SLE and impaired hippocampal neurogenesis. Although increased cell proliferation in the SVZ has been previously reported in other SLE models- MRL/MpJ mice and MRL/MpJ-Fas ${ }^{l p r}$ mice (Baker et al., 2006; 
Table 1

Summary showing the change of different markers- DCX, IBA-1 and GFAP in different brain regions of the diseased mice when compared with the pre-diseased mice in the present study

\begin{tabular}{lccc}
\hline & DCX & IBA-1 & GFAP \\
\hline Subventicular zone & $\uparrow$ & $?$ & $?$ \\
Corpus Callosum & $\uparrow$ & $\uparrow$ & $\uparrow$ \\
Hippocampus & $\downarrow$ & $?$ & $?$ \\
\hline
\end{tabular}

Abbreviations: $\uparrow$, increased; $\downarrow$, decreased; ?, haven't studied.

Stanojcic et al., 2009) (Table 2), to the best of our knowledge, an analysis of neuronal precursors in the corpus callosum has not been included in the previous studies. Under normal circumstances, new neurons generated in the SVZ migrate along the RMS to the OB (Altman, 1969; Kornack \& Rakic, 2001; Lois \& Alvarez-Buylla, 1994) and those neurons seldom reach the corpus callosum. Though the rare and abnormal migration of the new cells to the corpus callosum has been mentioned in other pathological conditions, e.g. stroke (Hua, Doucette, \& Walz, 2008; Osman, Porritt, Nilsson, \& Kuhn, 2011), our report of this phenomenon in the NZB/W F1 mice after the onset of SLE symptoms is novel. Our study is the first study showing the presence of neuronal precursor cells in the corpus callosum in the SLE animal model.

DCX is a microtubule-associated protein which is specifically expressed in migrating neuronal precursors (Couillard-Despres et al., 2005), and it is also a widely-used marker for neuronal precursor cells (Rao $\&$ Shetty, 2004) which could reflect the level of neurogenesis (Couillard-Despres et al., 2005). Since DCX has a critical role on neuronal migration (Koizumi et al., 2006) and the DCX+cells in our diseased mice exhibited the migrating-like morphology (elongated and leading processes) (Schaar \& McConnell, 2005; Wichterle, Garcia-Verdugo, \& Alvarez-Buylla, 1997), it was likely that the DCX+cells in the diseased mice of the present study were migrating. As the SVZ is the brain region where active neurogenesis takes place (Ming \& Song, 2005, 2011), it is possible that the DCX+cells were migrating from the SVZ to the corpus callosum after the onset of SLE symptoms. Since we could not find any DCX + cells in the cortex of the diseased mice, we deduced that the DCX + cells may have stopped migrating after they had reached the corpus callosum. At present, it is elusive why the abnormal migration was found in the corpus callosum after the onset of SLE symptoms. As different kinds of mood disorders, cognitive disorders and structural brain abnormalities were commonly found in the SLE patients (Appenzeller et al., 2006; Appenzeller et al., 2005; Jung et al., 2010; Stojanovich et al., 2007), it is worth further investigation on whether the altered migration of neuronal precursor cells has any relationship with those brain-related disorders.

Previous studies have reported that SLE results in inflammation in the brain (Crupi et al., 2010; Lapter et al., 2009). Activation of microglial cells and increased astrogliosis has been found in the hippocampus in different murine models of SLE, e.g. NZB/W F1 mice and BAFF transgenic mice (Crupi et al., 2010; Lapter et al., 2009). In the present study, after the onset of SLE symptoms, we have found the number of IBA-1 + cells and GFAP+ cells also increased in the corpus callosum of the female NZB/W F1 mice. In contrast to the finding in the hippocampus in the previous study (Crupi et al., 2010), in which hippocampal neurogenesis decreased with neuroinflammation exacerbated, our results showed that the corpus callosum of the diseased mice had the increase of neuronal precursors apart from the activation of microglia and astrocytes. The present finding suggests that neuroinflammation in SLE may not always suppress neurogenesis. According to literature, it has been shown that microglia could promote neurogenesis. Mouse microglial cells have been found to direct neural precursor cell migration and promote both adult and embryonic neural precursors to differentiate toward neuronal lineage (Aarum, Sandberg, Haeberlein, \& Persson, 2003). Another study also showed that microglia released neurotrophic factors and enhanced proliferation of neuronal precursor cells; it was suggested that the

Table 2

Summary showing the change of the markers-DCX and BrdU in different animal models of SLE

\begin{tabular}{|c|c|c|c|c|c|c|c|c|}
\hline & \multicolumn{2}{|c|}{$\begin{array}{c}\text { BAFF } \\
\text { (Crupi et al., 2010) } \\
\end{array}$} & \multicolumn{2}{|c|}{$\begin{array}{c}\text { MRL/MpJ } \\
\text { (Baker et al., 2006) } \\
\end{array}$} & \multicolumn{2}{|c|}{$\begin{array}{c}\text { MR/MpJ-Fas }{ }^{l p r} \\
\text { (Stanojcic et al., 2009) }\end{array}$} & \multicolumn{2}{|c|}{$\begin{array}{c}\text { NZB/WF1 } \\
\text { (The present study) }\end{array}$} \\
\hline & DCX & BrdU & DCX & BrdU & DCX & BrdU & DCX & BrdU \\
\hline Subventicular zone & $?$ & $?$ & + & $\uparrow$ & + & $\uparrow($ BrdU Protuberance) & $\uparrow$ & $?$ \\
\hline Corpus Callosum & $?$ & $?$ & $?$ & $?$ & $?$ & $?$ & $\uparrow$ & $?$ \\
\hline Hippocampus & $\downarrow$ & $\downarrow$ & $?$ & $?$ & + & $\uparrow$ & $\downarrow$ & $?$ \\
\hline
\end{tabular}

Abbreviations: $\uparrow$, increased; $\downarrow$, decreased; +, presence; ?, haven’t mentioned. 
proliferation was mediated by the activation of MAPK, PI3K/Akt and delta-Notch signaling pathways (Morgan, Taylor, \& Pocock, 2004). Apart from microglia, many studies also support the role of astrocytes in neurogenesis. Both in vivo and in vitro studies have found that astrocytes can promote migration of neural stem cells by releasing immunological factors, e.g. SDF- $1 \alpha$ (Imitola et al., 2004; Xu et al., 2007); furthermore, treatment of conditioned medium prepared from LPS-stimulated astrocytes could enhance proliferation and differentiation of neural stem cells (Wang et al., 2011). But for SLE, the possible relationship between neurogenesis and neuroinflammation remains unclear. There may be the possibility that IBA- $1+$ cells and GFAP+cells could more or less contribute to the neurogenesis after the onset of SLE symptoms in our SLE animal model, maybe by releasing some kind of immunological factors or growth factors that could promote neurogenesis. However, this could not be confirmed until further studies are performed.

To conclude, our study has found the abnormal increase of neuronal precursor cells in the corpus callosum of the female NZB/W F1 mice after the onset of SLE symptoms. The finding suggests that neurogenesis may be altered in this SLE model. As different manifestations of brain abnormalities and psychiatric symptoms were commonly found in the SLE patients (Appenzeller et al., 2006; Appenzeller et al., 2005; Jung et al., 2010; Stojanovich et al., 2007), further investigation of any functional consequence of altered neurogenesis in SLE models would be of interest.

\section{Acknowledgments}

The work is supported by Programme of Introducing Talents of Discipline to Universities (B14036), and Project of International, as well as Hong Kong, Macao \& Taiwan Science and Technology Cooperation Innovation Platform in Universities in Guangdong Province (2013gjhz0002). Support was also provided through grants to Jinan University Guangdong-Hong Kong-Macau Cooperation and Innovation Center for Tissue Regeneration and Repair.

\section{Conflict of interest}

The authors declare no conflicts of interest.

\section{References}

Aarum, J., Sandberg, K., Haeberlein, S.L.B., \& Persson, M.A.A. (2003). Migration and differentiation of neural precursor cells can be directed by microglia. Proceedings of the National Academy of Sciences of the United States of America, 100(26), 15983-15988.

Altman, J. (1969). Autoradiographic and histological studies of postnatal neurogenesis. IV. Cell proliferation and migration in the anterior forebrain, with special reference to persisting neurogenesis in the olfactory bulb. Journal of Comparative Neurology, 137(4), 433-457.

Alvarez-Buylla, A., \& Garcia-Verdugo, J.M. (2002). Neurogenesis in adult subventricular zone. The Journal of Neuroscience, 22(3), 629-634.

Appenzeller, S., Carnevalle, A.D., Li, L.M., Costallat, L.T., \& Cendes, F. (2006). Hippocampal atrophy in systemic lupus erythematosus. Annals of the Rheumatic Diseases, 65(12), 1585-1589.

Appenzeller, S., Rondina, J.M., Li, L.M., Costallat, L.T., \& Cendes, F. (2005). Cerebral and corpus callosum atrophy in systemic lupus erythematosus. Arthritis \& Rheumatology, 52(9), 2783-2789.

Arvidsson, A., Collin, T., Kirik, D., Kokaia, Z., \& Lindvall, O. (2002). Neuronal replacement from endogenous precursors in the adult brain after stroke. Nature Medicine, 8(9), 963-970.

Baker, K.L., Daniels, S.B., Lennington, J.B., Lardaro, T., Czap, A., Notti, R.Q., Cooper, O., Isacson, O., Frasca, S., Jr., \& Conover, J.C. (2006). Neuroblast protuberances in the subventricular zone of the regenerative MRL/MpJ mouse. Journal of Comparative Neurolology, 498(6), 747-761.

Ballok, D.A., Woulfe, J., Sur, M., Cyr, M., \& Sakic, B. (2004). Hippocampal damage in mouse and human forms of systemic autoimmune disease. Hippocampus, 14(5), 649-661.

Banati, R.B., Gehrmann, J., Schubert, P., \& Kreutzberg, G.W. (1993). Cytotoxicity of microglia. Glia, 7(1), 111-118.

Bonfanti, L., Peretto, P., Merighi, A., \& Fasolo, A. (1997). Newlygenerated cells from the rostral migratory stream in the accessory olfactory bulb of the adult rat. Neuroscience, 81(2), 489-502.

Brown, J.P., Couillard-Despres, S., Cooper-Kuhn, C.M., Winkler, J., Aigner, L., \& Kuhn, H.G. (2003). Transient expression of doublecortin during adult neurogenesis. Journal of Comparative Neurology, 467(1), 1-10.

Chao, C.C., Hu, S., Molitor, T.W., Shaskan, E.G., \& Peterson, P.K. (1992). Activated microglia mediate neuronal cell injury via a nitric oxide mechanism. The Journal of Immunology, 149(8), 2736-2741.

Chen, Y.L., Pan, A.W., Hsiung, P.C., \& Chung, L. (2015). Quality of Life Enhancement Programme for Individuals with Mood Disorder: A Randomized Controlled Pilot Study. Hong Kong Journal of Occupational Therapy, 25, 23-31.

Clelland, C.D., Choi, M., Romberg, C., Clemenson, G.D., Jr., Fragniere, A., Tyers, P., Jessberger, S., Saksida, L.M., Barker, R.A., Gage, F.H., \& Bussey, T.J. (2009). A functional role for adult hippocampal neurogenesis in spatial pattern separation. Science, 325(5937), 210-213.

Cooper, G.S., Parks, C.G., Treadwell, E.L., St Clair, E.W., Gilkeson, G.S., \& Dooley, M.A. (2004). Occupational risk factors 
for the development of systemic lupus erythematosus. The Journal of Rheumatology, 31(10), 1928-1933.

Couillard-Despres, S., Winner, B., Schaubeck, S., Aigner, R., Vroemen, M., Weidner, N., Bogdahn, U., Winkler, J., Kuhn, H.G., \& Aigner, L. (2005). Doublecortin expression levels in adult brain reflect neurogenesis. European Journal of Neuroscience, 21(1), 1-14.

Crupi, R., Cambiaghi, M., Spatz, L., Hen, R., Thorn, M., Friedman, E., Vita, G., \& Battaglia, F. (2010). Reduced adult neurogenesis and altered emotional behaviors in autoimmune-prone B-cell activating factor transgenic mice. Biological Psychiatry, 67(6), 558-566.

Curtis, M.A., Penney, E.B., Pearson, A.G., van Roon-Mom, W.M.C., Butterworth, N.J., Dragunow, M., Connor, B., \& Faull, R.L.M. (2003). Increased cell proliferation and neurogenesis in the adult human Huntington's disease brain. Proceedings of the National Academy of Sciences of the United States of America, 100(15), 9023-9027.

D’Cruz, D.P., Khamashta, M.A., \& Hughes, G.R. (2007). Systemic lupus erythematosus. Lancet, 369(9561), 587-596.

des Portes, V., Pinard, J.M., Billuart, P., Vinet, M.C., Koulakoff, A., Carrie, A., Gelot, A., Dupuis, E., Motte, J., Berwald-Netter, Y., Catala, M., Kahn, A., Beldjord, C., \& Chelly, J. (1998). A novel CNS gene required for neuronal migration and involved in X-linked subcortical laminar heterotopia and lissencephaly syndrome. Cell, 92(1), 51-61.

Dheen, S.T., Kaur, C., \& Ling, E.A. (2007). Microglial activation and its implications in the brain diseases. Current Medicinal Chemistry, 14(11), 1189-1197.

Eng, L.F., \& Ghirnikar, R.S. (1994). Gfap and Astrogliosis. Brain Pathology, 4(3), 229-237.

Eng, L.F., Yu, A.C.H., \& Lee, Y.L. (1992). Astrocytic Response to Injury. Progress in Brain Research, 94, 353-365.

Ge, S., Yang, C.H., Hsu, K.S., Ming, G.L., \& Song, H. (2007). A critical period for enhanced synaptic plasticity in newly generated neurons of the adult brain. Neuron, 54(4), 559-566.

Gehrmann, J., Matsumoto, Y., \& Kreutzberg, G.W. (1995). Microglia: Intrinsic immuneffector cell of the brain. Brain Research Reviews, 20(3), 269-287.

Gheusi, G., Cremer, H., McLean, H., Chazal, G., Vincent, J.D., \& Lledo, P.M. (2000). Importance of newly generated neurons in the adult olfactory bulb for odor discrimination. Proceedings of the National Academy of Sciences of the United States of America, 97(4), 1823-1828.

Gleeson, J.G., Lin, P.T., Flanagan, L.A., \& Walsh, C.A. (1999). Doublecortin is a microtubule-associated protein and is expressed widely by migrating neurons. Neuron, 23(2), 257271.

Hua, R., Doucette, R., \& Walz, W. (2008). Doublecortinexpressing cells in the ischemic penumbra of a small-vessel stroke. Journal of Neuroscience Research, 86(4), 883-893.

Imitola, J., Raddassi, K., Park, K.I., Mueller, F.J., Nieto, M., Teng, Y.D., Frenkel, D., Li, J., Sidman, R.L., Walsh, C.A., Snyder, E.Y., \& Khoury, S.J. (2004). Directed migration of neural stem cells to sites of CNS injury by the stromal cell-derived factor 1alpha/CXC chemokine receptor 4 pathway. Proceedings of the National Academy of Sciences of the United States of America, 101(52), 18117-18122.
Ito, D., Imai, Y., Ohsawa, K., Nakajima, K., Fukuuchi, Y., \& Kohsaka, S. (1998). Microglia-specific localisation of a novel calcium binding protein, Iba1. Molecular Brain Research, 57(1), 1-9.

Jung, R.E., Segall, J.M., Grazioplene, R.G., Qualls, C., Sibbitt, W.L., \& Roldan, C.A. (2010). Cortical thickness and subcortical gray matter reductions in neuropsychiatric systemic lupus erythematosus. PLoS One, 5(3), e9302.

Koizumi, H., Higginbotham, H., Poon, T., Tanaka, T., Brinkman, B.C., \& Gleeson, J.G. (2006). Doublecortin maintains bipolar shape and nuclear translocation during migration in the adult forebrain. Nature Neuroscience, 9(6), 779-786.

Kornack, D.R., \& Rakic, P. (2001). The generation, migration, and differentiation of olfactory neurons in the adult primate brain. Proceedings of the National Academy of Sciences of the United States of America, 98(8), 4752-4757.

Kowal, C., Degiorgio, L.A., Lee, J.Y., Edgar, M.A., Huerta, P.T., Volpe, B.T., \& Diamond, B. (2006). Human lupus autoantibodies against NMDA receptors mediate cognitive impairment. Proceedings of the National Academy of Sciences of the United States of America, 103(52), 19854-19859.

Lapter, S., Marom, A., Meshorer, A., Elmann, A., Sharabi, A., Vadai, E., Neufeld, A., Sztainberg, Y., Gil, S., Getselter, D., Chen, A., \& Mozes, E. (2009). Amelioration of Brain Pathology and Behavioral Dysfunction in Mice With Lupus Following Treatment With a Tolerogenic Peptide. Arthritis and Rheumatism, 60(12), 3744-3754.

Lau, B.W., Ren, C., Yang, J., Yan, S.W., Chang, R.C., Pu, M., \& So, K.F. (2011). Light deprivation induces depression-like behavior and suppresses neurogenesis in diurnal mongolian gerbil (Meriones unguiculatus). Cell Transplantation, 20(6), 871-881.

Lau, C.S., Yin, G., \& Mok, M.Y. (2006). Ethnic and geographical differences in systemic lupus erythematosus: An overview. Lupus, 15(11), 715-719.

Liu, J., Solway, K., Messing, R.O., \& Sharp, F.R. (1998). Increased neurogenesis in the dentate gyrus after transient global ischemia in gerbils. The Journal of Neuroscience, 18(19), 7768-7778

Lois, C., \& Alvarez-Buylla, A. (1994). Long-distance neuronal migration in the adult mammalian brain. Science, 264(5162), 1145-1148.

Manson, J.J., \& Isenberg, D.A. (2003). The pathogenesis of systemic lupus erythematosus. Netherlands Journal of Medicine, 61(11), 343-346.

Masuda, T., Isobe, Y., Aihara, N., Furuyama, F., Misumi, S., Kim, T.S., Nishino, H., \& Hida, H. (2007). Increase in neurogenesis and neuroblast migration after a small intracerebral hemorrhage in rats. Neuroscience Letters, 425(2), 114-119.

Ming, G.L., \& Song, H. (2005). Adult neurogenesis in the mammalian central nervous system. Annual Review of Neuroscience, 28, 223-250.

Ming, G.L., \& Song, H. (2011). Adult neurogenesis in the mammalian brain: Significant answers and significant questions. Neuron, 70(4), 687-702.

Morel, L., Croker, B.P., Blenman, K.R., Mohan, C., Huang, G., Gilkeson, G., \& Wakeland, E.K. (2000). Genetic reconstitution of systemic lupus erythematosus immunopathology with polycongenic murine strains. Proceedings of the National 
Academy of Sciences of the United States of America, 97(12), 6670-6675.

Morgan, S.C., Taylor, D.L., \& Pocock, J.M. (2004). Microglia release activators of neuronal proliferation mediated by activation of mitogen-activated protein kinase, phosphatidylinositol-3-kinase/Akt and delta-Notch signalling cascades. Journal of Neurochemistry, 90(1), 89-101.

Nacher, J., Crespo, C., \& McEwen, B.S. (2001). Doublecortin expression in the adult rat telencephalon. European Journal of Neuroscience, 14(4), 629-644.

Osman, A.M., Porritt, M.J., Nilsson, M., \& Kuhn, H.G. (2011). Long-term stimulation of neural progenitor cell migration after cortical ischemia in mice. Stroke, 42(12), 3559-3565.

Parent, J.M., Valentin, V.V., \& Lowenstein, D.H. (2002). Prolonged seizures increase proliferating neuroblasts in the adult rat subventricular zone-olfactory bulb pathway. The Journal of Neuroscience, 22(8), 3174-3188.

Parent, J.M., Vexler, Z.S., Gong, C., Derugin, N., \& Ferriero, D.M. (2002). Rat forebrain neurogenesis and striatal neuron replacement after focal stroke. Annals of Neurology, 52(6), 802-813.

Parks, C.G., Cooper, G.S., Nylander-French, L.A., Sanderson, W.T., Dement, J.M., Cohen, P.L., Dooley, M.A., Treadwell, E.L., St Clair, E.W., Gilkeson, G.S., Hoppin, J.A., \& Savitz, D.A. (2002). Occupational exposure to crystalline silica and risk of systemic lupus erythematosus: A population-based, case-control study in the southeastern United States. Arthritis \& Rheumatology, 46(7), 1840-1850.

Perry, D., Sang, A., Yin, Y., Zheng, Y.Y., \& Morel, L. (2011). Murine models of systemic lupus erythematosus. Journal of Biomedicine and Biotechnology, 2011, 271694.

Rao, M.S., \& Shetty, A.K. (2004). Efficacy of doublecortin as a marker to analyse the absolute number anddendritic growth of newly generated neurons in the adult dentate gyrus. European Journal of Neuroscience, 19(2), 234-246.

Ruan, L., Lau, B.W., Wang, J., Huang, L., Zhuge, Q., Wang, B., Jin, K., \& So, K.F. (2014). Neurogenesis in neurological and psychiatric diseases and brain injury: From bench to bedside. Progress in Neurobiology, 115, 116-137.

Schaar, B.T., \& McConnell, S.K. (2005). Cytoskeletal coordination during neuronal migration. Proceedings of the National Academy of Sciences of the United States of America, 102(38), 13652-13657.
Sestak, A.L., Furnrohr, B.G., Harley, J.B., Merrill, J.T., \& Namjou, B. (2011). The genetics of systemic lupus erythematosus and implications for targeted therapy. Annals of the Rheumatic Diseases, 70(Suppl 1), i37-43.

Smith, M.T., Pencea, V., Wang, Z., Luskin, M.B., \& Insel, T.R. (2001). Increased number of BrdU-labeled neurons in the rostral migratory stream of the estrous prairie vole. Hormone and Behavior, 39(1), 11-21.

Stanojcic, M., Burstyn-Cohen, T., Nashi, N., Lemke, G., \& Sakic, B. (2009). Disturbed distribution of proliferative brain cells during lupus-like disease. Brain, Behavior and Immunity, 23(7), 1003-1013.

Stewart, V.C., Sharpe, M.A., Clark, J.B., \& Heales, S.J.R. (2000). Astrocyte-derived nitric oxide causes both reversible and irreversible damage to the neuronal mitochondrial respiratory chain. Journal of Neurochemistry, 75(2), 694-700.

Stojanovich, L., Zandman-Goddard, G., Pavlovich, S., \& Sikanich, N. (2007). Psychiatric manifestations in systemic lupus erythematosus. Autoimmunity Reviews, 6(6), 421-426.

Sundholm-Peters, N.L., Yang, H.K., Goings, G.E., Walker, A.S., \& Szele, F.G. (2005). Subventricular zone neuroblasts emigrate toward cortical lesions. Journal of Neuropathology \& Experimental Neurology, 64(12), 1089-1100.

Tani, M., Glabinski, A.R., Tuohy, V.K., Stoler, M.H., Estes, M. L., \& Ransohoff, R.M. (1996). In situ hybridization analysis of glial fibrillary acidic protein mRNA reveals evidence of biphasic astrocyte activation during acute experimental autoimmune encephalomyelitis. American Journal of Pathology, 148(3), 889-896.

Wang, F.W., Hao, H.B., Zhao, S.D., Zhang, Y.M., Liu, Q., Liu, H.J., Liu, S.M., Yuan, Q.H., Bing, L.J., Ling, E.A., \& Hao, A.J. (2011). Roles of activated astrocyte in neural stem cell proliferation and differentiation. Stem Cell Research, 7(1), 41-53.

Wichterle, H., Garcia-Verdugo, J.M., \& Alvarez-Buylla, A. (1997). Direct evidence for homotypic, glia-independent neuronal migration. Neuron, 18(5), 779-791.

Xu, Q., Wang, S., Jiang, X., Zhao, Y., Gao, M., Zhang, Y., Wang, X., Tano, K., Kanehara, M., Zhang, W., \& Ishida, T. (2007). Hypoxia-induced astrocytes promote the migration of neural progenitor cells via vascular endothelial factor, stem cell factor, stromal-derived factor-1alpha and monocyte chemoattractant protein-1 upregulation in vitro. Clinical and Experimental Pharmacology and Physiology, 34(7), 624-631. 